\title{
Fusion for the one-dimensional Hubbard model
}

\author{
Niklas Beisert ${ }^{1}$, Marius de Leeuw ${ }^{1,2}$ and Panchali NaG ${ }^{1}$ \\ 1 Institut für Theoretische Physik, \\ Eidgenössische Technische Hochschule Zürich \\ Wolfgang-Pauli-Strasse 27, 8093 Zürich, Switzerland \\ 2 Niels Bohr Institute, \\ Copenhagen University \\ Blegdamsvej 17, 2100 Copenhagen Ø, Denmark \\ nbeisert@itp.phys.ethz.ch, deleeuwm@nbi.ku.dk, nagp@student.ethz.ch

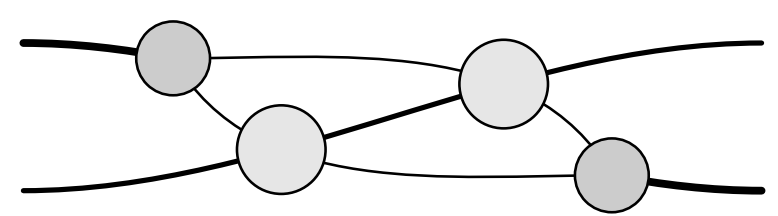

\begin{abstract}
We discuss a formulation of the fusion procedure for integrable models which is suitable for application to non-standard R-matrices. It allows for construction of bound state R-matrices for AdS/CFT worldsheet scattering or equivalently for the one-dimensional Hubbard model. We also discuss some peculiar cases that arise in these models.
\end{abstract}




\section{Introduction}

Integrable systems constitute a special class of physical models that are exactly solvable [1]. The key ingredient that allows for the explicit construction of exact solutions is the so-called R-matrix. For most known integrable models, the corresponding R-matrices are determined by the underlying symmetry algebra of the system. This is usually an infinite-dimensional Hopf algebra of Yangian or quantum affine type.

Computing the R-matrix in various representations of such an algebra then describes different types of particles. For example, the Heisenberg XXX model has the Yangian of $\mathfrak{s u}(2)$ as its symmetry algebra. The R-matrix in the fundamental representation simply describes a chain of spin- $\frac{1}{2}$ particles. Similarly, particles of higher spin can be considered by taking higher-dimensional representations of $\mathfrak{s u}(2)$.

On the other hand, from basic representation theory it is well known that for example the tensor product of two spin- $\frac{1}{2}$ particles splits into a spin- 1 and a spin-0 representation. In other words, one should be able to relate R-matrices in higher dimensional representations to the fundamental R-matrix. For example, the R-matrix of spin-1 particles should allow for some decomposition into the R-matrix of spin- $\frac{1}{2}$ particles. Similarly, from the fundamental representation it should be possible to construct new R-matrices corresponding to other representations. This construction goes under the name of fusion [2]. For most integrable systems this procedure is well-understood, however, the established formulas do not directly apply for some of the more exotic integrable models.

Recently, there was renewed interest in the field of integrable systems due to their appearance in string and gauge theory via the AdS/CFT correspondence ( see [3] and references therein). In particular, this integrable structure gave rise to an unusual $\mathrm{R}$ matrix that displays Yangian symmetry corresponding to the centrally extended $\mathfrak{s u}(2 \mid 2)$ Lie superalgebra [4,5]. Remarkably, this R-matrix turned out to be directly related to Shastry's R-matrix $|6|$ describing the one-dimensional Hubbard model [7].

It soon became clear that for a full description of the string model, the R-matrices in higher dimensional representations corresponding to bound states were needed [8]. These bound state R-matrices could be computed directly by invoking the Yang-Baxter equation $\overline{[9]}$ or by using Yangian symmetry [10]. However, how to obtain these matrices directly from the fundamental R-matrix was unknown since the usual fusion procedure breaks down. In this note we will introduce a slight generalization of the fusion procedure for integrable models which allows us to obtain bound state R-matrices for the AdS/CFT S-matrix and Shastry's R-matrix.

This paper is organized as follows. In Section 2 we introduce our fusion procedure and study it at the level of R-matrices. Then in Section 3 we study more advanced aspects of it. Afterwards we turn to applications: In Section 4 we discuss the well-known example of the XXX spin chain before moving on to the novel case of AdS/CFT worldsheet scattering and the one-dimensional Hubbard model in Section 5.

\section{Fusion}

Consider an integrable system whose fundamental degrees of freedom are described by an $n$-dimensional vector space $\mathbb{V}^{\mathrm{F}}$. These might represent the spin degrees of freedom of an integrable spin chain or the particle flavors of an integrable scattering problem in $1+1$ 
dimensions. Their interactions are described by an $\left(n^{2} \times n^{2}\right)$-dimensional R-matrix

$$
\mathcal{R}\left(u_{1}, u_{2}\right): \mathbb{V}^{\mathrm{F}} \otimes \mathbb{V}^{\mathrm{F}} \rightarrow \mathbb{V}^{\mathrm{F}} \otimes \mathbb{V}^{\mathrm{F}}
$$

where the parameters $u_{1}, u_{2} \in \mathrm{M}^{\mathrm{F}}$ describe the inhomogeneities of the spin sites or the particle rapidities. The space of parameters $\mathrm{M}^{\mathrm{F}}$ is typically a one-dimensional complex manifold, ${ }^{1}$ such as the complex plane or the Riemann sphere with certain punctures.

For an integrable system, the R-matrix satisfies the Yang-Baxter equation and the involution property (the latter relation is understood up to an overall factor)

$$
\mathcal{R}_{12} \mathcal{R}_{13} \mathcal{R}_{23}=\mathcal{R}_{23} \mathcal{R}_{13} \mathcal{R}_{12}, \quad \mathcal{R}_{12} \mathcal{R}_{21} \sim 1_{12}
$$

Here and in the following, the indices denote the spaces in a tensor product as well as the associated parameters $u_{k}$.

\subsection{Singularities}

Suppose that there are pairs of points

$$
u_{\langle 12\rangle}=\left(u_{1}, u_{2}\right) \in \mathrm{M}^{\mathrm{B}} \subset \mathrm{M}^{\mathrm{F}} \times \mathrm{M}^{\mathrm{F}}
$$

where the R-matrix becomes non-invertible. ${ }^{2}$ In other words, the rank of the R-matrix drops below its maximum

$$
\operatorname{rank} \mathcal{R}\left(u_{1}, u_{2}\right)=m<n^{2} .
$$

Commonly, these points form a one-dimensional sub-manifold ${ }^{3} M^{B}$ of $M^{F} \times M^{F}$. The point $u_{\langle 12\rangle} \in \mathrm{M}^{\mathrm{B}}$ can thus be treated as a continuous parameter, and it is on the same footing as the parameters $u_{k} \in \mathrm{M}^{\mathrm{F}}$. In scattering theory, such singular behavior signals the existence of composite particles which are naturally part of the physical scattering problem. $\cdot{ }^{4}$ For these we will define new R-matrices to be interpreted as scattering matrices involving composite particles. We can thus extend the integrable system by adding these composite particles as additional degrees of freedom.

More precisely, we are led to the introduction of an $m$-dimensional vector space $\mathbb{V}^{\mathrm{B}}$ by 'fusing' two spaces $\mathbb{V}^{\mathrm{F}}$ such that the extended integrable system is defined on $\mathbb{V}^{\text {ext }}:=$ $\mathbb{V}^{\mathrm{F}} \oplus \mathbb{V}^{\mathrm{B}}$. The corresponding R-matrix can be schematically written in block form

$$
\mathcal{R}^{\text {ext }}: \mathbb{V}^{\text {ext }} \otimes \mathbb{V}^{\text {ext }} \rightarrow \mathbb{V}^{\text {ext }} \otimes \mathbb{V}^{\text {ext }}, \quad \mathcal{R}^{\text {ext }}=\operatorname{diag}\left(\mathcal{R}^{\mathrm{FF}}, \mathcal{R}^{\mathrm{FB}}, \mathcal{R}^{\mathrm{BF}}, \mathcal{R}^{\mathrm{BB}}\right),
$$

where the various blocks are maps of the types $\left(A_{i}=\mathrm{F}, \mathrm{B}\right)$

$$
\mathcal{R}^{A_{1} A_{2}}\left(u_{1}, u_{2}\right): \mathbb{V}^{A_{1}} \otimes \mathbb{V}^{A_{2}} \rightarrow \mathbb{V}^{A_{1}} \otimes \mathbb{V}^{A_{2}}, \quad\left(u_{1}, u_{2}\right) \in \mathrm{M}^{A_{1}} \times \mathrm{M}^{A_{2}} .
$$

\footnotetext{
${ }^{1}$ Additional continuous parameters or even discrete parameters are conceivable as well.

${ }^{2}$ The R-matrix can have singular points where some of its eigenvalues diverge. As we are not interested in overall factors of the R-matrix (which may well depend on $u_{1}$ and $u_{2}$ ) we should rescale the R-matrix at these points to remove the singularity. In other words, what counts is that the leading contribution in a Laurent expansion is non-invertible.

${ }^{3}$ Discrete points with this property can also be considered along the same lines. We will, however, mostly be interested in the case of continuous values for $u_{\langle 12\rangle}$.

${ }^{4}$ In fact, they are only part of the physical problem if they are also bound. The distinction between bound and unbound composite particles makes no difference here, and we shall refer to them collectively as composite states.
} 
In particular, $\mathcal{R}^{\mathrm{FF}} \equiv \mathcal{R}$. The fact that $\mathcal{R}^{\mathrm{ext}}$ satisfies the Yang-Baxter equation is then equivalent to the statement

$$
\mathcal{R}_{12}^{A_{1} A_{2}} \mathcal{R}_{13}^{A_{1} A_{3}} \mathcal{R}_{23}^{A_{2} A_{3}}=\mathcal{R}_{23}^{A_{2} A_{3}} \mathcal{R}_{13}^{A_{1} A_{3}} \mathcal{R}_{12}^{A_{1} A_{2}} .
$$

Moreover, the R-matrices $\mathcal{R}^{A_{1} A_{2}}$ have the involution property

$$
\mathcal{R}_{12}^{A_{1} A_{2}} \mathcal{R}_{21}^{A_{2} A_{1}} \sim 1_{12}^{A_{1} A_{2}}
$$

Of course the fusion procedure can be recursively applied, leading to larger and larger integrable systems.

\section{$2.2 \quad$ Procedure}

In the following, we shall construct the fused R-matrices and afterwards check their properties.

Embedding and fusion matrices. We consider the R-matrix $\mathcal{R}\left(u_{\langle 12\rangle}\right):=\mathcal{R}\left(u_{1}, u_{2}\right)$ at a point $u_{\langle 12\rangle}$ where the rank drops to $m<n^{2}$. At this point we can decompose it as a product of three matrices

$$
\begin{aligned}
& \mathcal{E}\left(u_{\langle 12\rangle}\right): \mathbb{V}^{\mathrm{B}} \rightarrow \mathbb{V}^{\mathrm{F}} \otimes \mathbb{V}^{\mathrm{F}}, \\
& \mathcal{H}\left(u_{\langle 12\rangle}\right): \mathbb{V}^{\mathrm{B}} \rightarrow \mathbb{V}^{\mathrm{B}}, \\
& \mathcal{F}\left(u_{\langle 12\rangle}\right): \mathbb{V}^{\mathrm{F}} \otimes \mathbb{V}^{\mathrm{F}} \rightarrow \mathbb{V}^{\mathrm{B}},
\end{aligned}
$$

with the properties

$$
\mathcal{R}\left(u_{\langle 12\rangle}\right)=\mathcal{E}\left(u_{\langle 12\rangle}\right) \mathcal{H}\left(u_{\langle 12\rangle}\right) \mathcal{F}\left(u_{\langle 12\rangle}\right), \quad \mathcal{F}\left(u_{\langle 12\rangle}\right) \mathcal{E}\left(u_{\langle 12\rangle}\right)=1^{\mathrm{B}}, \quad \mathcal{H} \text { invertible. }
$$

Here, $\mathcal{F}$ is a (surjective) $m \times n^{2}$ matrix which fuses the tensor product $\mathbb{V}^{\mathrm{F}} \otimes \mathbb{V}^{\mathrm{F}}$ to the space $\mathbb{V}^{\mathrm{B}}$, and $\mathcal{E}$ is an (injective) $n^{2} \times m$ matrix which embeds $\mathbb{V}^{\mathrm{B}}$ into $\mathbb{V}^{\mathrm{F}} \otimes \mathbb{V}^{\mathrm{F}}$. For convenience, we assume these two matrices to be pseudo-inverses as in $(2.10)$. Finally, $\mathcal{H}$ is a (bijective) $m \times m$ matrix and it represents the action of $\mathcal{R}$ on the space $\mathbb{V}^{\mathrm{B}}$. In the following we shall describe two ways of obtaining the decomposition.

First of all, let $\mathbb{V}^{\mathrm{B}}$ be the image of $\mathcal{R}$. Thus $\mathcal{R}$ can be understood as a map $\mathbb{V}^{\mathrm{F}} \otimes$ $\mathbb{V}^{\mathrm{F}} \rightarrow \mathbb{V}^{\mathrm{B}}$. Furthermore, define $\mathcal{E}$ as the trivial embedding map $\mathbb{V}^{\mathrm{B}} \rightarrow \mathbb{V}^{\mathrm{F}} \otimes \mathbb{V}^{\mathrm{F}}$. The above decomposition can be obtained as $\mathcal{F}=(\mathcal{R E})^{-1} \mathcal{R}$ and $\mathcal{H}=\mathcal{R E}$. Note that this construction requires $\mathcal{H}=\mathcal{R} \mathcal{E}$ to be invertible. This combination is not invertible precisely if the image of $\mathcal{R}$ contains a vector that is in the kernel of $\mathcal{R}$. In other words, the map $\mathcal{R}$ contains a non-trivial nilpotent part. This case is more difficult to handle, and we shall exclude it for the time being. Later on in Sec. 5.3, we shall discuss an explicit example.

An alternative construction of the decomposition uses eigenvectors where we assume that the Jordan decomposition is trivial (i.e. the nilpotent case is excluded). The matrix $\mathcal{R}$ possesses $m$ non-zero eigenvalues $\lambda_{1}, \ldots, \lambda_{m}$. Let $e_{1}, \ldots, e_{m}$ denote the associated right eigenvectors of $\mathcal{R}$ and $e^{1}, \ldots, e^{m}$ the left eigenvectors

$$
\mathcal{R} e_{k}=\lambda_{k} e_{k}, \quad e^{k} \mathcal{R}=\lambda_{k} e^{k} .
$$

All of these quantities are functions of $u_{\langle 12\rangle}$. We normalize the vectors such that they form two dual bases for the space $\mathbb{V}^{\mathrm{B}}$

$$
e^{k} e_{l}=\delta_{l}^{k}, \quad \mathcal{R}=\sum_{k} \lambda_{k} e_{k} e^{k} .
$$



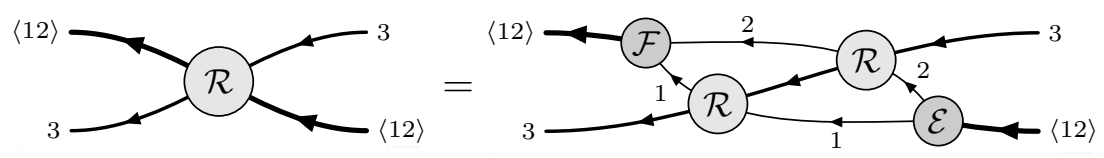

Figure 1: Diagrammatical representation of fusion.

We then define $\mathcal{E}, \mathcal{H}$ and $\mathcal{F}$ as the matrices of eigenvectors and eigenvalues

$$
\mathcal{E}=\left(\begin{array}{llll}
e_{1} & e_{2} & \ldots & e_{m}
\end{array}\right), \quad \mathcal{H}=\operatorname{diag}\left(\lambda_{1}, \ldots, \lambda_{m}\right), \quad \mathcal{F}=\left(\begin{array}{llll}
e^{1} & e^{2} & \ldots & e^{m}
\end{array}\right)^{\top} .
$$

By their definition $\mathcal{E}$ and $\mathcal{F}$ satisfy the relations $(2.10)$.

The relations (2.10) imply the following useful identities

$$
\begin{aligned}
\mathcal{E}\left(u_{\langle 12\rangle}\right) \mathcal{H}\left(u_{\langle 12\rangle}\right) & =\mathcal{R}\left(u_{\langle 12\rangle}\right) \mathcal{E}\left(u_{\langle 12\rangle}\right), \\
\mathcal{H}\left(u_{\langle 12\rangle}\right) \mathcal{F}\left(u_{\langle 12\rangle}\right) & =\mathcal{F}\left(u_{\langle 12\rangle}\right) \mathcal{R}\left(u_{\langle 12\rangle}\right), \\
\mathcal{R}\left(u_{\langle 12\rangle}\right) \mathcal{E}\left(u_{\langle 12\rangle}\right) \mathcal{F}\left(u_{\langle 12\rangle}\right) & =\mathcal{R}\left(u_{\langle 12\rangle}\right) .
\end{aligned}
$$

These are the crucial relations that enable us to carry out the fusion procedure.

Fused R-matrices. We introduce R-matrices by using $\mathcal{E}, \mathcal{F}$ to 'fuse' together two spaces $\mathbb{V}_{1}^{\mathrm{F}} \otimes \mathbb{V}_{2}^{\mathrm{F}}$ into $\mathbb{V}_{\langle 12\rangle}^{\mathrm{B}}$ with $u_{\langle 12\rangle}=\left(u_{1}, u_{2}\right)$. In particular, we are led to (cf. Fig. 1)

$$
\begin{aligned}
& \mathcal{R}_{\langle 12\rangle 3}^{\mathrm{BF}}\left(u_{\langle 12\rangle}, u_{3}\right):=\mathcal{F}_{\langle 12\rangle}\left(u_{\langle 12\rangle}\right) \mathcal{R}_{13}\left(u_{1}, u_{3}\right) \mathcal{R}_{23}\left(u_{2}, u_{3}\right) \mathcal{E}_{\langle 12\rangle}\left(u_{\langle 12\rangle}\right), \\
& \mathcal{R}_{1\langle 23\rangle}^{\mathrm{FB}}\left(u_{1}, u_{\langle 23\rangle}\right):=\mathcal{F}_{\langle 23\rangle}\left(u_{\langle 23\rangle}\right) \mathcal{R}_{13}\left(u_{1}, u_{3}\right) \mathcal{R}_{12}\left(u_{1}, u_{2}\right) \mathcal{E}_{\langle 23\rangle}\left(u_{\langle 23\rangle}\right) .
\end{aligned}
$$

Any state from $\mathbb{V}_{\langle 12\rangle}^{\mathrm{B}} \otimes \mathbb{V}_{3}^{\mathrm{F}}$ is mapped to $\mathbb{V}_{1}^{\mathrm{F}} \otimes \mathbb{V}_{2}^{\mathrm{F}} \otimes \mathbb{V}_{3}^{\mathrm{F}}$ by $\mathcal{E}$, acted upon with $\mathcal{R}$ and then mapped back by $\mathcal{F}$.

Notice the different ordering of the R-matrices which is needed when spaces two and three are fused rather than spaces one and two. The R-matrix $\mathcal{R}^{\mathrm{BB}}$ can then be defined by applying the fusion procedure twice

$$
\mathcal{R}_{\langle 12\rangle\langle 34\rangle}^{\mathrm{BB}}\left(u_{\langle 12\rangle}, u_{\langle 34\rangle}\right):=\mathcal{F}_{\langle 34\rangle}\left(u_{\langle 34\rangle}\right) \mathcal{R}_{\langle 12\rangle}^{\mathrm{BF}}\left(u_{\langle 12\rangle}, u_{4}\right) \mathcal{R}_{\langle 12\rangle}^{\mathrm{BF}}\left(u_{\langle 12\rangle}, u_{3}\right) \mathcal{E}_{\langle 34\rangle}\left(u_{\langle 34\rangle}\right) .
$$

In particular, from $(2.14)$ it is readily seen that $(2.17)$ can be cast into the symmetric form

$$
\mathcal{R}_{\langle 12\rangle\langle 34\rangle}:=\mathcal{F}_{\langle 12\rangle} \mathcal{F}_{\langle 34\rangle} \mathcal{R}_{14} \mathcal{R}_{24} \mathcal{R}_{13} \mathcal{R}_{23} \mathcal{E}_{\langle 12\rangle} \mathcal{E}_{\langle 34\rangle},
$$

which demonstrates that it is independent of the order of fusing the underlying spaces. Here and in the following, we drop the parameters $u$ and the labels F, B in favor of a more concise presentation. They can be recovered from the labels of the associated spaces.

\subsection{Relations}

In order to show that these R-matrices indeed describe an integrable system we have to show that they are invertible and that they satisfy the Yang-Baxter equation. 
Involution property. We have to show the involution property (2.8) which reads more explicitly

$$
\begin{aligned}
\mathcal{R}_{\langle 12\rangle 3}^{\mathrm{BF}}\left(u_{\langle 12\rangle}, u_{3}\right) \mathcal{R}_{3\langle 12\rangle}^{\mathrm{FB}}\left(u_{3}, u_{\langle 12\rangle}\right) & \sim 1, \\
\mathcal{R}_{\langle 12\rangle\langle 34\rangle}^{\mathrm{BB}}\left(u_{\langle 12\rangle}, u_{\langle 34\rangle}\right) \mathcal{R}_{\langle 34\rangle\langle 12\rangle}^{\mathrm{BB}}\left(u_{\langle 34\rangle}, u_{\langle 12\rangle}\right) & \sim 1 .
\end{aligned}
$$

Let us prove the first instance. For conciseness we will omit the arguments. Furthermore, we shall put brackets around the terms to be transformed in the next step

$$
\begin{aligned}
\mathcal{H}_{\langle 12\rangle} \mathcal{R}_{\langle 12\rangle} \mathcal{R}_{3\langle 12\rangle} & =\left[\mathcal{H}_{\langle 12\rangle} \mathcal{F}_{\langle 12\rangle}\right] \mathcal{R}_{13} \mathcal{R}_{23} \mathcal{E}_{\langle 12\rangle} \mathcal{F}_{\langle 12\rangle} \mathcal{R}_{32} \mathcal{R}_{31} \mathcal{E}_{\langle 12\rangle} \\
& =\mathcal{F}_{\langle 12\rangle}\left[\mathcal{R}_{12} \mathcal{R}_{13} \mathcal{R}_{23}\right] \mathcal{E}_{\langle 12\rangle} \mathcal{F}_{\langle 12\rangle} \mathcal{R}_{32} \mathcal{R}_{31} \mathcal{E}_{\langle 12\rangle} \\
& =\mathcal{F}_{\langle 12\rangle} \mathcal{R}_{23} \mathcal{R}_{13}\left[\mathcal{R}_{12} \mathcal{E}_{\langle 12\rangle} \mathcal{F}_{\langle 12\rangle}\right] \mathcal{R}_{32} \mathcal{R}_{31} \mathcal{E}_{\langle 12\rangle} \\
& =\mathcal{F}_{\langle 12\rangle}\left[\mathcal{R}_{23} \mathcal{R}_{13} \mathcal{R}_{12}\right] \mathcal{R}_{32} \mathcal{R}_{31} \mathcal{E}_{\langle 12\rangle} \\
& =\left[\mathcal{F}_{\langle 12\rangle} \mathcal{R}_{12}\right]\left[\mathcal{R}_{13}\left[\mathcal{R}_{23} \mathcal{R}_{32}\right] \mathcal{R}_{31}\right] \mathcal{E}_{\langle 12\rangle} \\
& \sim \mathcal{H}_{\langle 12\rangle}\left[\mathcal{F}_{\langle 12\rangle} \mathcal{E}_{\langle 12\rangle}\right] \\
& =\mathcal{H}_{\langle 12\rangle} .
\end{aligned}
$$

Invertibility of $\mathcal{H}$ then gives the desired result. In general, the strategy is to remove intermediate factors of $\mathcal{E}$ and $\mathcal{F}$ by use of the Yang-Baxter equation $(2.2)$ and the properties $(2.14)$.

Yang-Baxter equation. Subsequently, it also follows directly from $(2.14)$ that the above introduced R-matrices $(2.15)-(2.17)$ satisfy the various versions of the Yang-Baxter equation outlined in $(2.7)$.

For example, since $\mathcal{R}$ satisfies the Yang-Baxter equation (2.2) itself, $(2.14)$ yields (we again suppress the explicit arguments)

$$
\begin{aligned}
\mathcal{H}_{\langle 12\rangle} \mathcal{R}_{\langle 12\rangle} \mathcal{R}_{\langle 12\rangle} \mathcal{R}_{34} & =\left[\mathcal{H}_{\langle 12\rangle} \mathcal{F}_{\langle 12\rangle}\right] \mathcal{R}_{13} \mathcal{R}_{23} \mathcal{E}_{\langle 12\rangle} \mathcal{F}_{\langle 12\rangle} \mathcal{R}_{14} \mathcal{R}_{24}\left[\mathcal{E}_{\langle 12\rangle} \mathcal{R}_{34}\right] \\
& =\mathcal{F}_{\langle 12\rangle}\left[\mathcal{R}_{12} \mathcal{R}_{13} \mathcal{R}_{23} \mathcal{E}_{\langle 12\rangle}\right] \mathcal{F}_{\langle 12\rangle} \mathcal{R}_{14} \mathcal{R}_{24} \mathcal{R}_{34} \mathcal{E}_{\langle 12\rangle} \\
& =\mathcal{F}_{\langle 12\rangle} \mathcal{R}_{12}\left[\mathcal{R}_{13} \mathcal{R}_{23} \mathcal{R}_{14} \mathcal{R}_{24} \mathcal{R}_{34}\right] \mathcal{E}_{\langle 12\rangle} \\
& =\left[\mathcal{F}_{\langle 12\rangle} \mathcal{R}_{12} \mathcal{R}_{34}\right] \mathcal{R}_{14} \mathcal{R}_{24} \mathcal{R}_{13} \mathcal{R}_{23} \mathcal{E}_{\langle 12\rangle} \\
& =\mathcal{R}_{34} \mathcal{F}_{\langle 12\rangle}\left[\mathcal{R}_{12} \mathcal{R}_{14} \mathcal{R}_{24}\right] \mathcal{R}_{13} \mathcal{R}_{23} \mathcal{E}_{\langle 12\rangle} \\
& =\mathcal{R}_{34}\left[\mathcal{F}_{\langle 12\rangle} \mathcal{R}_{12}\right] \mathcal{R}_{14} \mathcal{R}_{24} \mathcal{E}_{\langle 12\rangle} \mathcal{F}_{\langle 12\rangle} \mathcal{R}_{13} \mathcal{R}_{23} \mathcal{E}_{\langle 12\rangle} \\
& =\left[\mathcal{R}_{34} \mathcal{H}_{\langle 12\rangle}\right]\left[\mathcal{F}_{\langle 12\rangle} \mathcal{R}_{14} \mathcal{R}_{24} \mathcal{E}_{\langle 12\rangle}\right]\left[\mathcal{F}_{\langle 12\rangle} \mathcal{R}_{13} \mathcal{R}_{23} \mathcal{E}_{\langle 12\rangle}\right] \\
& =\mathcal{H}_{\langle 12\rangle} \mathcal{R}_{34} \mathcal{R}_{\langle 12\rangle} \mathcal{R}_{\langle 12\rangle 3}
\end{aligned}
$$

This proves that the R-matrices $(2.15)-(2.17)$ indeed describe the scattering of a new (composite) type of particle in this model.

\section{$3 \quad$ Further properties}

We have established the basic features of fused R-matrices. In the following we will discuss further properties. 


\subsection{Algebra}

Suppose there is a Hopf algebra $H$ describing the symmetries of our integrable system. In particular, the R-matrix, by definition, intertwines the coproduct and opposite coproduct in the representation $\rho^{\mathrm{F}}(u): \mathrm{H} \rightarrow \mathbb{V}^{\mathrm{F}}$ under which our fundamental degrees of freedom transform, i.e. for any generator $\mathbb{J} \in \mathrm{H}$

$$
\left(\rho_{1}^{\mathrm{F}}\left(u_{1}\right) \otimes \rho_{2}^{\mathrm{F}}\left(u_{2}\right)\right)\left[\Delta^{\mathrm{op}}(\mathbb{J})\right] \mathcal{R}_{12}\left(u_{1}, u_{2}\right)=\mathcal{R}_{12}\left(u_{1}, u_{2}\right)\left(\rho_{1}^{\mathrm{F}}\left(u_{1}\right) \otimes \rho_{2}^{\mathrm{F}}\left(u_{2}\right)\right)[\Delta(\mathbb{J})] .
$$

We define a new representation $\rho^{\mathrm{B}}\left(u_{\langle 12\rangle}\right): \mathrm{H} \rightarrow \mathbb{V}^{\mathrm{B}}$ for the composite degrees of freedom

$$
\rho_{\langle 12\rangle}^{\mathrm{B}}\left(u_{\langle 12\rangle}\right)[\mathbb{J}]:=\mathcal{F}_{\langle 12\rangle}\left(u_{\langle 12\rangle}\right)\left(\rho_{1}^{\mathrm{F}}\left(u_{1}\right) \otimes \rho_{2}^{\mathrm{F}}\left(u_{2}\right)\right)[\Delta(\mathbb{J})] \mathcal{E}_{\langle 12\rangle}\left(u_{\langle 12\rangle}\right) .
$$

We will refer to this as the fused or composite representation. This representation clearly is $m$-dimensional.

Let us show that $(\overline{3.2})$ indeed defines a representation by proving that it respects the multiplicative structure. We have from $(2.14)$ and the cocommutativity $(3.1)$ of the coproduct, that for any $\mathbb{J}, \mathbb{J}^{\prime} \in \mathrm{H}$

$$
\begin{aligned}
\mathcal{H}_{\langle 12\rangle} \rho_{\langle 12\rangle}[\mathbb{J}] \rho_{\langle 12\rangle}\left[\mathbb{J}^{\prime}\right] & =\mathcal{F}_{\langle 12\rangle} \mathcal{R}_{12}\left(\rho_{1} \otimes \rho_{2}\right)[\Delta(\mathbb{J})] \mathcal{E}_{\langle 12\rangle} \mathcal{F}_{\langle 12\rangle}\left(\rho_{1} \otimes \rho_{2}\right)\left[\Delta\left(\mathbb{J}^{\prime}\right)\right] \mathcal{E}_{\langle 12\rangle} \\
& =\mathcal{F}_{\langle 12\rangle}\left(\rho_{1} \otimes \rho_{2}\right)\left[\Delta^{\mathrm{op}}(\mathbb{J})\right] \mathcal{R}_{12} \mathcal{E}_{\langle 12\rangle} \mathcal{F}_{\langle 12\rangle}\left(\rho_{1} \otimes \rho_{2}\right)\left[\Delta\left(\mathbb{J}^{\prime}\right)\right] \mathcal{E}_{\langle 12\rangle} \\
& =\mathcal{F}_{\langle 12\rangle}\left(\rho_{1} \otimes \rho_{2}\right)\left[\Delta^{\mathrm{op}}(\mathbb{J})\right] \mathcal{R}_{12}\left(\rho_{1} \otimes \rho_{2}\right)\left[\Delta\left(\mathbb{J}^{\prime}\right)\right] \mathcal{E}_{\langle 12\rangle} \\
& =\mathcal{F}_{\langle 12\rangle} \mathcal{R}_{12}\left(\rho_{1} \otimes \rho_{2}\right)[\Delta(\mathbb{J})]\left(\rho_{1} \otimes \rho_{2}\right)\left[\Delta\left(\mathbb{J}^{\prime}\right)\right] \mathcal{E}_{\langle 12\rangle} \\
& =\mathcal{H}_{\langle 12\rangle} \mathcal{F}_{\langle 12\rangle}\left(\rho_{1} \otimes \rho_{2}\right)\left[\Delta\left(\mathbb{J} \mathbb{J}^{\prime}\right)\right] \mathcal{E}_{\langle 12\rangle} \\
& =\mathcal{H}_{\langle 12\rangle} \rho_{\langle 12\rangle}\left[\mathbb{J} \mathbb{J}^{\prime}\right] .
\end{aligned}
$$

Furthermore, the R-matrices (2.15) $-(2.17)$ naturally intertwine the coproduct in the new representation. Explicitly,

$$
\left(\rho_{1}^{A_{1}} \otimes \rho_{2}^{A_{2}}\right)\left[\Delta^{\mathrm{op}}(\mathbb{J})\right] \mathcal{R}_{12}^{A_{1} A_{2}}=\mathcal{R}_{12}^{A_{1} A_{2}}\left(\rho_{1}^{A_{1}} \otimes \rho_{2}^{A_{2}}\right)[\Delta(\mathbb{J})] .
$$

For instance, let us prove the intertwining relation for the case $A_{1}=\mathrm{B}, A_{2}=\mathrm{F}$. This is most conveniently done in the Sweedler notation $\Delta(\mathbb{I})=\sum \mathbb{J}_{(1)} \otimes \mathbb{J}_{(2)}$. Co-associativity of the Hopf algebra is then written as

$$
\sum \mathbb{J}_{(1)(1)} \otimes \mathbb{J}_{(1)(2)} \otimes \mathbb{J}_{(2)}=\sum \mathbb{J}_{(1)} \otimes \mathbb{J}_{(2)(1)} \otimes \mathbb{J}_{(2)(2)}
$$

and the intertwining property $(\overline{3.1})$ of the R-matrix is formulated as

$$
\sum \mathcal{R}_{12} \rho_{1}\left[\mathbb{J}_{(1)}\right] \rho_{2}\left[\mathbb{J}_{(2)}\right]=\sum \rho_{1}\left[\mathbb{J}_{(2)}\right] \rho_{2}\left[\mathbb{J}_{(1)}\right] \mathcal{R}_{12}
$$

In this language, we have (for conciseness we suppress the arguments $u, v$ of the R-matrices and the sums)

$$
\begin{aligned}
\mathcal{R}_{\langle 12\rangle 3}\left(\rho_{\langle 12\rangle} \otimes \rho_{3}\right)[\Delta(\mathbb{J})] & =\mathcal{R}_{\langle 12\rangle} \mathcal{F}_{\langle 12\rangle} \rho_{1}\left[\mathbb{J}_{(1)}\right] \rho_{2}\left[\mathbb{J}_{(2)(1)}\right] \rho_{3}\left[\mathbb{J}_{(2)(2)}\right] \mathcal{E}_{\langle 12\rangle} \\
& =\mathcal{F}_{\langle 12\rangle} \mathcal{R}_{13} \mathcal{R}_{23} \rho_{1}\left[\mathbb{J}_{(1)}\right] \rho_{2}\left[\mathbb{J}_{(2)(1)}\right] \rho_{3}\left[\mathbb{J}_{(2)(2)}\right] \mathcal{E}_{\langle 12\rangle} \\
& =\mathcal{F}_{\langle 12\rangle} \rho_{1}\left[\mathbb{J}_{(2)(1)}\right] \rho_{2}\left[\mathbb{J}_{(2)(2)}\right] \rho_{3}\left[\mathbb{J}_{(1)}\right] \mathcal{E}_{\langle 12\rangle} \mathcal{F}_{\langle 12\rangle} \mathcal{R}_{13} \mathcal{R}_{23} \mathcal{E}_{\langle 12\rangle} \\
& =\left(\rho_{\langle 12\rangle} \otimes \rho_{3}\right)\left[\Delta^{\mathrm{op}}(\mathbb{J})\right] \mathcal{R}_{\langle 12\rangle},
\end{aligned}
$$

where we used (2.14) repeatedly and (3.6) in the third step. This proves that $\mathcal{R}^{\mathrm{BF}}$ displays the expected cocommutativity properties. 


\subsection{Auxiliary features}

Here we will discuss some auxiliary features of the fused R-matrices.

Similarity transformations. We have the freedom to apply a similarity transformation $W\left(u_{\langle 12\rangle}\right)$ to the space $\mathbb{V}^{\mathrm{B}}$

$$
\begin{aligned}
\mathcal{E}\left(u_{\langle 12\rangle}\right) & \rightarrow \mathcal{E}\left(u_{\langle 12\rangle}\right) W\left(u_{\langle 12\rangle}\right)^{-1} \\
\mathcal{F}\left(u_{\langle 12\rangle}\right) & \rightarrow W\left(u_{\langle 12\rangle}\right) \mathcal{F}\left(u_{\langle 12\rangle}\right) \\
\mathcal{H}\left(u_{\langle 12\rangle}\right) & \rightarrow W\left(u_{\langle 12\rangle}\right) \mathcal{H}\left(u_{\langle 12\rangle}\right) W\left(u_{\langle 12\rangle}\right)^{-1}
\end{aligned}
$$

This transformation affects none of the relations $(2.10)$, and therefore all the above results apply to the transformed system without further ado. In the construction of $\mathcal{E}, \mathcal{H}, \mathcal{F}$ via eigenvectors (2.13), the similarity transformation does not preserve the diagonal nature of $\mathcal{H}$ (unless $W$ is diagonal as well). However, we have not made explicit use of this property in the constructions.

Opposite form. A similarity transformation by $\mathcal{H}_{\langle 12\rangle}$ has a curious effect on the fused R-matrices

$$
\begin{aligned}
\mathcal{H}_{\langle 12\rangle} \mathcal{R}_{\langle 12\rangle 3} \mathcal{H}_{\langle 12\rangle}^{-1} & =\mathcal{H}_{\langle 12\rangle} \mathcal{F}_{\langle 12\rangle} \mathcal{R}_{13} \mathcal{R}_{23} \mathcal{E}_{\langle 12\rangle} \mathcal{H}_{\langle 12\rangle}^{-1} \\
& =\mathcal{F}_{\langle 12\rangle} \mathcal{R}_{12} \mathcal{R}_{13} \mathcal{R}_{23} \mathcal{E}_{\langle 12\rangle} \mathcal{H}_{\langle 12\rangle}^{-1} \\
& =\mathcal{F}_{\langle 12\rangle} \mathcal{R}_{23} \mathcal{R}_{13} \mathcal{R}_{12} \mathcal{E}_{\langle 12\rangle} \mathcal{H}_{\langle 12\rangle}^{-1} \\
& =\mathcal{F}_{\langle 12\rangle} \mathcal{R}_{23} \mathcal{R}_{13} \mathcal{E}_{\langle 12\rangle} .
\end{aligned}
$$

Compared to the original definition $\mathcal{R}_{\langle 12\rangle 3}=\mathcal{F}_{\langle 12\rangle} \mathcal{R}_{13} \mathcal{R}_{23} \mathcal{E}_{\langle 12\rangle}$, we observe that conjugation by $\mathcal{H}_{\langle 12\rangle}$ flips the order of the R-matrix factors within the fused R-matrix.

This observation goes hand in hand with the definition (3.2) of the fused representation via the coproduct. If instead of the coproduct we use the opposite coproduct, the resulting representation is related to the original one by a simple similarity transformation

$$
\mathcal{F}_{\langle 12\rangle}\left(\rho_{1} \otimes \rho_{2}\right)[\Delta(\mathbb{J})] \mathcal{E}_{\langle 12\rangle}=\mathcal{H}_{\langle 12\rangle}^{-1} \mathcal{F}_{\langle 12\rangle}\left(\rho_{1} \otimes \rho_{2}\right)\left[\Delta^{\mathrm{op}}(\mathbb{J})\right] \mathcal{E}_{\langle 12\rangle} \mathcal{H}_{\langle 12\rangle}
$$

As usual we used (2.14) and the intertwining property of the R-matrix.

Symmetric R-matrices. In many practical applications $\mathcal{R}\left(u_{\langle 12\rangle}\right)$, acting as an operator on the space $\mathbb{V}^{\mathrm{F}} \otimes \mathbb{V}^{\mathrm{F}}$, is symmetric w.r.t. some inner product, e.g. the standard inner product $\langle a, b\rangle:=a^{\top} b$ defined on $\mathbb{V}^{\mathrm{F}}$. In most cases, $\mathcal{R}\left(u_{\langle 12\rangle}\right)=\mathcal{R}\left(u_{\langle 12\rangle}\right)^{\top}$ admits an orthonormal basis of eigenvectors. ${ }^{5}$ In addition, the fusion and embedding matrices are conjugate to each other $\mathcal{F}=\mathcal{E}^{\top}$.

One minor problem is that the resulting R-matrices $(2.15)$ are not symmetric. Transposition reverses the order of the constituent R-matrices which corresponds to a similarity transformation according to $(3.9)$

$$
\mathcal{R}_{\langle 12\rangle 3}^{\top}=\mathcal{F}_{\langle 12\rangle} \mathcal{R}_{23} \mathcal{R}_{13} \mathcal{E}_{\langle 12\rangle}=\mathcal{H}_{\langle 12\rangle} \mathcal{R}_{\langle 12\rangle 3} \mathcal{H}_{12}^{-1}
$$

\footnotetext{
${ }^{5}$ This is evident if the inner product is positive definite. For indefinite inner products (including complex symmetric matrices), eigenvectors can be null. In this case the eigenvectors cannot be normalized, and even a non-trivial Jordan decomposition may arise.
} 
By applying a similarity transformation (3.8) defined by a $W$ such that $\mathcal{H}=W^{\top} W$, the resulting $\mathrm{R}$-matrices become symmetric

$$
\mathcal{R}_{\langle 12\rangle 3}^{\prime \top}=\left(W \mathcal{R}_{\langle 12\rangle 3} W^{-1}\right)^{\top}=W^{-\top} \mathcal{H}_{\langle 12\rangle} \mathcal{R}_{\langle 12\rangle 3} \mathcal{H}_{\langle 12\rangle}^{-1} W^{\top}=\left(W^{-\top} \mathcal{R}_{\langle 12\rangle 3}^{\top} W^{\top}\right)^{\top}=\mathcal{R}_{\langle 12\rangle 3}^{\prime}
$$

\subsection{Complementary fusion}

A fused R-matrix can also be defined for the complement $\mathbb{V}_{\langle\overline{12}\rangle}^{\overline{\mathrm{B}}}$ of the space $\mathbb{V}_{\langle 12\rangle}^{\mathrm{B}}$

$$
\mathbb{V}_{1}^{\mathrm{F}} \otimes \mathbb{V}_{2}^{\mathrm{F}}=\mathbb{V}_{\langle 12\rangle}^{\mathrm{B}} \oplus \mathbb{V}_{\langle\overline{12}\rangle}^{\overline{\mathrm{B}}}, \quad \mathcal{R}_{\langle\overline{12}\rangle 3}^{\overline{\mathrm{B}} \mathrm{F}}: \mathbb{V}_{\langle\overline{12}\rangle}^{\overline{\mathrm{B}}} \otimes \mathbb{V}_{3}^{\mathrm{F}} \rightarrow \mathbb{V}_{\langle\overline{12}\rangle}^{\overline{\mathrm{B}}} \otimes \mathbb{V}_{3}^{\mathrm{F}}
$$

As we shall see, this space is even better suited for fusion.

Complementary space. To define an R-matrix for the complement, we need to define fusion and embedding matrices for the complementary space

$$
\overline{\mathcal{E}}\left(u_{\langle 12\rangle}\right): \mathbb{V}^{\overline{\mathrm{B}}} \rightarrow \mathbb{V}^{\mathrm{F}} \otimes \mathbb{V}^{\mathrm{F}}, \quad \overline{\mathcal{F}}\left(u_{\langle 12\rangle}\right): \mathbb{V}^{\mathrm{F}} \otimes \mathbb{V}^{\mathrm{F}} \rightarrow \mathbb{V}^{\overline{\mathrm{B}}} .
$$

They are defined to obey the following orthogonality properties with the original embedding and fusion matrices in (3.15):

$$
\overline{\mathcal{F}}\left(u_{\langle 12\rangle}\right) \overline{\mathcal{E}}\left(u_{\langle 12\rangle}\right)=1^{\overline{\mathrm{B}}}, \quad \mathcal{F}\left(u_{\langle 12\rangle}\right) \overline{\mathcal{E}}\left(u_{\langle 12\rangle}\right)=0, \quad \overline{\mathcal{F}}\left(u_{\langle 12\rangle}\right) \mathcal{E}\left(u_{\langle 12\rangle}\right)=0
$$

They directly imply the completeness relations

$$
\mathcal{E}_{\langle 12\rangle} \mathcal{F}_{\langle 12\rangle}+\overline{\mathcal{E}}_{\langle\overline{12}\rangle} \overline{\mathcal{F}}_{\langle\overline{12\rangle}}=1_{12} .
$$

as well as orthogonality relations with the R-matrix

$$
\mathcal{R}\left(u_{\langle 12\rangle}\right) \overline{\mathcal{E}}\left(u_{\langle 12\rangle}\right)=0, \quad \overline{\mathcal{F}}\left(u_{\langle 12\rangle}\right) \mathcal{R}\left(u_{\langle 12\rangle}\right)=0
$$

Complementary R-matrix. One can define a complementary R-matrix in analogy to (2.15)

$$
\mathcal{R}_{\langle\overline{\mathrm{B}}\rangle 3}^{\overline{\mathrm{B}}}\left(u_{\langle 12\rangle}, u_{3}\right):=\overline{\mathcal{F}}_{\langle\overline{12\rangle}}\left(u_{\langle 12\rangle}\right) \mathcal{R}_{13}\left(u_{1}, u_{3}\right) \mathcal{R}_{23}\left(u_{2}, u_{3}\right) \overline{\mathcal{E}}_{\langle\overline{12\rangle}}\left(u_{\langle 12\rangle}\right) .
$$

The other related R-matrices follow in a similar fashion. The various integrability relationships can be derived in a similar fashion to the above. The general strategy is to remove the factors of $\overline{\mathcal{E}}_{\langle\overline{12}\rangle} \overline{\mathcal{F}}_{\langle\overline{12}\rangle}$ which typically appear between the various R-matrices. The starting point is the relationship $\mathcal{F}_{\langle 12\rangle} \mathcal{R}_{13} \mathcal{R}_{23} \overline{\mathcal{E}}_{\langle\overline{12}\rangle}=0$ which follows from the above definitions

$$
\mathcal{H}_{\langle 12\rangle} \mathcal{F}_{\langle 12\rangle} \mathcal{R}_{13} \mathcal{R}_{23} \overline{\mathcal{E}}_{\langle\overline{12\rangle}}=\mathcal{F}_{\langle 12\rangle} \mathcal{R}_{12} \mathcal{R}_{13} \mathcal{R}_{23} \overline{\mathcal{E}}_{\langle\overline{12}\rangle}=\mathcal{F}_{\langle 12\rangle} \mathcal{R}_{23} \mathcal{R}_{13} \mathcal{R}_{12} \overline{\mathcal{E}}_{\langle\overline{12}\rangle}=0
$$

Together with the completeness relationship (3.16) one can show

$$
\overline{\mathcal{E}}_{\langle\overline{12}\rangle} \overline{\mathcal{F}}_{\langle\overline{12}\rangle} \mathcal{R}_{13} \mathcal{R}_{23} \overline{\mathcal{E}}_{\langle\overline{12}\rangle}=\mathcal{R}_{13} \mathcal{R}_{23} \overline{\mathcal{E}}_{\langle\overline{12}\rangle}-\mathcal{E}_{\langle 12\rangle} \mathcal{F}_{\langle 12\rangle} \mathcal{R}_{13} \mathcal{R}_{23} \overline{\mathcal{E}}_{\langle\overline{12}\rangle}=\mathcal{R}_{13} \mathcal{R}_{23} \overline{\mathcal{E}}_{\langle\overline{12}\rangle}
$$

Therefore, all intermediate factors of $\overline{\mathcal{E}}_{\langle\overline{12}\rangle} \overline{\mathcal{F}}_{\langle\overline{12}\rangle}$ can be removed iteratively from products of R-matrices from the left to the right. 
Similarly, one can prove that

$$
\overline{\mathcal{F}}_{\langle\overline{12}\rangle} \mathcal{R}_{23} \mathcal{R}_{13} \mathcal{E}_{\langle 12\rangle}=0, \quad \mathcal{E}_{\langle 12\rangle} \mathcal{F}_{\langle 12\rangle} \mathcal{R}_{23} \mathcal{R}_{13} \mathcal{E}_{\langle 12\rangle}=\mathcal{R}_{23} \mathcal{R}_{13} \mathcal{E}_{\langle 12\rangle}
$$

On the level of the R-matrix this simply corresponds to a similarity transformation on the composite particle space applied to the original fused R-matrix (3.9).

Put differently, the combinations $\mathcal{R}_{13} \mathcal{R}_{23}$ and $\mathcal{R}_{23} \mathcal{R}_{13}$, when viewed as a block diagonal matrix, effectively possess a triangular shape:

$$
\begin{aligned}
& \mathcal{R}_{13} \mathcal{R}_{23}:\left\{\begin{array}{l}
\mathbb{V}_{\langle 12\rangle}^{\mathrm{B}} \otimes \mathbb{V}_{3}^{\mathrm{F}} \rightarrow\left(\mathbb{V}_{\langle 12\rangle}^{\mathrm{B}} \oplus \mathbb{V}_{\langle\overline{12}\rangle}^{\overline{\mathrm{B}}}\right) \otimes \mathbb{V}_{3}^{\mathrm{F}}, \\
\mathbb{V}_{\langle\overline{\mathrm{B}}\rangle}^{\overline{\mathrm{B}}} \otimes \mathbb{V}_{3}^{\mathrm{F}} \rightarrow \mathbb{V}_{\langle 1 \overline{\mathrm{B}}\rangle}^{\overline{\mathrm{B}}} \otimes \mathbb{V}_{3}^{\mathrm{F}} .
\end{array}\right. \\
& \mathcal{R}_{23} \mathcal{R}_{13}:\left\{\begin{array}{l}
\mathbb{V}_{\langle 12\rangle}^{\mathrm{B}} \otimes \mathbb{V}_{3}^{\mathrm{F}} \rightarrow \mathbb{V}_{\langle 12\rangle}^{\mathrm{B}} \otimes \mathbb{V}_{3}^{\mathrm{F}}, \\
\mathbb{V}_{\langle\overline{1}\rangle}^{\overline{\mathrm{B}}} \otimes \mathbb{V}_{3}^{\mathrm{F}} \rightarrow\left(\mathbb{V}_{\langle 12\rangle}^{\mathrm{B}} \oplus \mathbb{V}_{\langle\overline{12}\rangle}^{\overline{\mathrm{B}}}\right) \otimes \mathbb{V}_{3}^{\mathrm{F}} .
\end{array}\right.
\end{aligned}
$$

Composite states from the complementary subspace $\mathbb{V}_{\langle\overline{12}\rangle}^{\overline{\mathrm{B}}}$ are mapped to the same subspace by $\mathcal{R}_{13} \mathcal{R}_{23}$. Conversely, composite states from the original subspace $\mathbb{V}_{\langle 12\rangle}^{\mathrm{B}}$ can map to both subspaces. Fusion as defined in (2.15) works only due to the presence of the projector $\mathcal{F}_{\langle 12\rangle}$. However, for the opposite R-matrix (3.9) this reverses and states from $\mathbb{V}_{\langle 12\rangle}^{\mathrm{B}}$ are mapped to the same subspace. This is at the cost of a similarity transformation on $\mathbb{V}_{\langle 12\rangle}^{\mathrm{B}}$.

Opposite fusion. The involution property $(2.2)$ also has an interesting implication on the complementary space $\mathbb{V}^{\overline{\mathrm{B}}}$ which we shall discuss in the following. According to our assumptions, the first $m$ eigenvalues of $\mathcal{R}\left(u_{\langle 12\rangle}\right)$ are non-zero while the other $n^{2}-m$ vanish at the point $u_{\langle 12\rangle}=\left(u_{1}, u_{2}\right)$. We assume that the latter fall off linearly in $\epsilon$ when approaching the singular point as in $\left(u_{1}, u_{2}+\epsilon\right) \rightarrow u_{\langle 12\rangle}$. Furthermore, also the product $\mathcal{R}_{12} \mathcal{R}_{21}$ is assumed to be proportional to $\epsilon$.

By the involution property $(2.2)$ we have that away from the singular points $\mathcal{R}_{12}$ is invertible with inverse proportional to $\mathcal{R}_{21}$. Therefore $\mathcal{R}_{12}$ and $\mathcal{R}_{21}$ share the same eigenvectors, but with inverse eigenvalues. Now, by our assumptions on the behavior near the singular point, we are led to the conclusion that the first $m$ eigenvalues of $\mathcal{R}_{21}$ fall off linearly with $m$ while the remaining $n^{2}-m$ eigenvalues approach a constant ${ }^{6}$

Therefore, the null space of $\mathcal{R}_{21}\left(u_{\langle 21\rangle}\right)$ at $u_{\langle 21\rangle}:=\left(u_{2}, u_{1}\right)$ is given by the image of $\mathcal{E}_{\langle 12\rangle}\left(u_{\langle 12\rangle}\right)$ whereas the non-trivial eigenspace is the image of $\mathcal{E}_{\langle 21\rangle}\left(u_{\langle 21\rangle}\right)$. Consequently, the space $\mathbb{V}_{\langle 21\rangle}^{\overline{\mathrm{B}}}$ is the complement of $\mathbb{V}_{\langle 12\rangle}^{\mathrm{B}}$ within $\mathbb{V}_{1}^{\mathrm{F}} \otimes \mathbb{V}_{2}^{\mathrm{F}}$, and it has dimension $\bar{m}:=$ $n^{2}-m$. We therefore reproduce the elements of complementary fusion

$$
\mathbb{V}_{\langle 21\rangle}^{\overline{\mathrm{B}}}=\mathbb{V}_{\langle\overline{12}\rangle}^{\overline{\mathrm{B}}}, \quad \mathcal{F}_{\langle 21\rangle} \sim \overline{\mathcal{F}}_{\langle\overline{12}\rangle}, \quad \mathcal{E}_{\langle 21\rangle} \sim \overline{\mathcal{E}}_{\langle\overline{12}\rangle} .
$$

All the constructions for the complementary fused states and operators proceed as before with the roles of spaces 1 and 2 interchanged.

\subsection{Algebraic Bethe ansatz}

Let us briefly touch upon the effect of fusion on monodromy and transfer matrices that play a central role in the algebraic Bethe Ansatz.

\footnotetext{
${ }^{6}$ If some of the eigenvalues of $\mathcal{R}_{12}$ scale with a higher power of $\epsilon$ (consequently also $\mathcal{R}_{12} \mathcal{R}_{21}$ ), only fewer than $n^{2}-m$ eigenvalues will be finite. The following considerations would have to be adapted accordingly. See Sec. 5.3 for a concrete example of this case.
} 
RTT-relation. The key relation in the algebraic Bethe ansatz is the so-called RTTrelation. The RTT-relation describes the commutation relations between the elements of an $n \times n$ dimensional operator valued matrix $\mathcal{T}^{\mathrm{F}}(u): \mathbb{V}^{\mathrm{F}} \rightarrow \mathbb{V}^{\mathrm{F}} \otimes \mathcal{O}$, called the monodromy matrix. We have

$$
\mathcal{R}_{12}^{\mathrm{FF}}\left(u_{1}, u_{2}\right) \mathcal{T}_{1}^{\mathrm{F}}\left(u_{1}\right) \mathcal{T}_{2}^{\mathrm{F}}\left(u_{2}\right)=\mathcal{T}_{2}^{\mathrm{F}}\left(u_{2}\right) \mathcal{T}_{1}^{\mathrm{F}}\left(u_{1}\right) \mathcal{R}_{12}^{\mathrm{FF}}\left(u_{1}, u_{2}\right)
$$

However, rather than taking $\mathcal{R}_{12}^{\mathrm{FF}}\left(u_{1}, u_{2}\right)$ one can also consider taking fused $\mathrm{R}$-matrices and consider the RTT relation this would generate. To this end, we introduce a fused monodromy matrix

$$
\mathcal{T}_{\langle 12\rangle}^{\mathrm{B}}\left(u_{\langle 12\rangle}\right):=\mathcal{F}_{\langle 12\rangle}\left(u_{\langle 12\rangle}\right) \mathcal{T}_{1}^{\mathrm{F}}\left(u_{1}\right) \mathcal{T}_{2}^{\mathrm{F}}\left(u_{2}\right) \mathcal{E}_{\langle 12\rangle}\left(u_{\langle 12\rangle}\right)
$$

It is straightforward to show that $\mathcal{T}_{\langle 12\rangle}^{\mathrm{B}}$ satisfies the RTT-relation for fused R-matrices.

An object of special interest is the transfer matrix, which is defined as the trace of the monodromy matrix

$$
t^{\mathrm{F}}(u)=\operatorname{tr}_{\mathrm{F}} \mathcal{T}^{\mathrm{F}}(u), \quad \quad t^{\mathrm{B}}\left(u_{\langle 12\rangle}\right)=\operatorname{tr}_{\mathrm{B}} \mathcal{T}^{\mathrm{B}}\left(u_{\langle 12\rangle}\right) .
$$

This object generates the mutually commuting set of operators that is the defining property of integrable systems.

Fusion for transfer matrices. We can formulate the relation between the transfer matrices in different representations. Consider the product of two transfer matrices and use the completeness relation $(3.16)$

$$
\begin{aligned}
t^{\mathrm{F}}\left(u_{1}\right) t^{\mathrm{F}}\left(u_{2}\right) & =\operatorname{tr}_{12}\left[\mathcal{T}_{1}\left(u_{1}\right) \mathcal{T}_{2}\left(u_{2}\right)\right] \\
& =\operatorname{tr}_{\langle 12\rangle}\left[\mathcal{F}_{\langle 12\rangle} \mathcal{T}_{1}\left(u_{1}\right) \mathcal{T}_{2}\left(u_{2}\right) \mathcal{E}_{\langle 12\rangle}\right]+\operatorname{tr}_{\langle 21\rangle}\left[\mathcal{F}_{\langle 21\rangle} \mathcal{T}_{1}\left(u_{1}\right) \mathcal{T}_{2}\left(u_{2}\right) \mathcal{E}_{\langle 21\rangle}\right] \\
& =t^{\mathrm{B}}\left(u_{\langle 12\rangle}\right)+t^{\overline{\mathrm{B}}}\left(u_{\langle 21\rangle}\right)
\end{aligned}
$$

For the latter term, we note that a similarity transformation by $\mathcal{H}_{\langle 21\rangle}$ is required to interchange the order of monodromy matrices in analogy to $(\overline{3.9})$.

\section{The Heisenberg XXX spin chain}

To illustrate our fusion procedure, let us first consider the Heisenberg XXX spin chain. The fundamental particles transform in the spin- $\frac{1}{2}$ representation of $\mathfrak{s u}(2)$. The corresponding R-matrix is of difference form $\mathcal{R}_{\mathrm{XXX}}\left(u_{1}, u_{2}\right)=\mathcal{R}_{\mathrm{XXX}}\left(u_{1}-u_{2}\right)$ and is given by

$$
\mathcal{R}_{\mathrm{XXX}}(u)=\left(\begin{array}{cccc}
u+1 & 0 & 0 & 0 \\
0 & u & 1 & 0 \\
0 & 1 & u & 0 \\
0 & 0 & 0 & u+1
\end{array}\right)
$$

There are two points at which the rank of $\mathcal{R}_{\mathrm{Xxx}}(u)$ is not maximal, namely at $u= \pm 1$. At $u=1$ the rank is three, while at $u=-1$ the rank is one. These points correspond to the singlet and triplet that arise in the decomposition of the tensor product of two spin- $\frac{1}{2}$ representations. 
Singlet. At the point $u_{1}-u_{2}=-1$, there is only one eigenvector with non-zero eigenvalue and our projector is

$$
\mathcal{E}=\mathcal{F}^{\top}=\frac{1}{\sqrt{2}}\left(\begin{array}{c}
0 \\
1 \\
-1 \\
0
\end{array}\right), \quad \mathcal{H}=-2 .
$$

It is easy to check that the identities from (2.14) hold. The representation that is generated according to $(\overline{3.2})$ is the trivial representation. Consequently, the fused R-matrix describing the scattering of a singlet state with a fundamental particle is given by

$$
\mathcal{R}_{\langle 12\rangle 3}\left(u_{\langle 12\rangle}, u_{3}\right)=\left[\left(u_{2}-u_{3}\right)^{2}-1\right]\left(\begin{array}{ll}
1 & 0 \\
0 & 1
\end{array}\right) .
$$

In other words, we see that the singlet has trivial scattering with a doublet (up to an overall factor which depends on the definition of the overall factor of $\mathcal{R}_{\mathrm{Xxx}}$ ).

Triplet. The other point $u_{1}-u_{2}=+1$ is the opposite of $u_{1}-u_{2}=-1$. The resulting space therefore is the complement of the above singlet. More concretely, there is only one null vector and from the three remaining eigenvectors we find

$$
\mathcal{E}=\mathcal{F}^{\top}=\left(\begin{array}{ccc}
1 & 0 & 0 \\
0 & \frac{1}{\sqrt{2}} & 0 \\
0 & \frac{1}{\sqrt{2}} & 0 \\
0 & 0 & 1
\end{array}\right), \quad \mathcal{H}=\operatorname{diag}(2,2,2)
$$

It is easily seen that this will give rise to the usual spin-1 representation of $\mathfrak{s u}(2)$. Indeed, (3.2) yields for the simple roots

$$
\rho_{\langle 12\rangle}\left[\mathbb{S}^{+}\right]=\left(\begin{array}{ccc}
0 & \sqrt{2} & 0 \\
0 & 0 & \sqrt{2} \\
0 & 0 & 0
\end{array}\right), \quad \rho_{\langle 12\rangle}\left[\mathbb{S}^{-}\right]=\left(\begin{array}{ccc}
0 & 0 & 0 \\
\sqrt{2} & 0 & 0 \\
0 & \sqrt{2} & 0
\end{array}\right) .
$$

Consequently, we recover the standard composite state R-matrix $\mathcal{R}_{\langle 12\rangle 3}\left(u_{\langle 12\rangle}, u_{3}\right)$ for the triplet-doublet case from (2.15)

$$
\left(u_{1}-u_{3}\right)\left(\begin{array}{cccccc}
u_{2}-u_{3}+2 & 0 & 0 & 0 & 0 & 0 \\
0 & u_{2}-u_{3} & \sqrt{2} & 0 & 0 & 0 \\
0 & \sqrt{2} & u_{2}-u_{3}+1 & 0 & 0 & 0 \\
0 & 0 & 0 & u_{2}-u_{3}+1 & \sqrt{2} & 0 \\
0 & 0 & 0 & \sqrt{2} & u_{2}-u_{3} & 0 \\
0 & 0 & 0 & 0 & 0 & u_{2}-u_{3}+2
\end{array}\right) .
$$

One can now easily check that the Yang-Baxter equation holds.

\section{The Hubbard model}

The R-matrix for the Hubbard model is best described in terms of its symmetry algebra; centrally extended $\mathfrak{s u}(2 \mid 2)$. This algebra is obtained from regular $\mathfrak{s u}(2 \mid 2)$ by adjoining 
two additional central charges to it. The R-matrix in the fundamental representation is completely fixed by the intertwining property $(3.1)[4,11]$. The R-matrices involving composite state representations are fixed by Yangian invariance [10].

It turns out that there are two cases where the R-matrix becomes of lower rank. There are two cases where it becomes of rank 8 and the fused representation is a (a)symmetric short representation. Moreover, there is a point where the R-matrix reduces to rank 1 corresponding to a singlet representation.

We will show that the bound state R-matrices found in the literature $[9]$ follow from our fusion procedure.

\subsection{The Hubbard model R-matrix}

The R-matrix for the Hubbard model is a $4^{2} \times 4^{2}$ matrix. It acts on the tensor product of two 2|2-dimensional spaces with bosonic basis vectors $\left|\phi^{a}\right\rangle, a=1,2$ and their fermionic counterparts $\left|\psi^{\alpha}\right\rangle, \alpha=3,4$. For convenience let us introduce the $2 \mid 2$-dimensional basis vector $E^{A}=\left(\left|\phi^{1}\right\rangle,\left|\phi^{2}\right\rangle,\left|\psi^{3}\right\rangle,\left|\psi^{4}\right\rangle\right)$ and let $E_{B}^{A}$ be the matrix unities with a $(-1)^{|B|}$ in row $A$ and column $B$. The fundamental R-matrix is then of the form

$$
\mathcal{R}\left(u_{1}, u_{2}\right)=(-1)^{|B|+|C|} E^{A}{ }_{B} \otimes E^{C}{ }_{D} \mathcal{R}^{B}{ }_{A}{ }^{D}{ }_{C}\left(u_{1}, u_{2}\right)
$$

with the only non-zero entries given by

$$
\begin{aligned}
& \mathcal{R}^{a}{ }_{b d}{ }^{c}=\delta_{d}^{a} \delta_{b}^{c}+\left(\delta_{b}^{a} \delta_{d}^{c}-\delta_{d}^{a} \delta_{b}^{c}\right) \frac{x_{1}^{+}-x_{2}^{+}}{x_{1}^{-}-x_{2}^{+}} \frac{x_{1}^{-}}{x_{1}^{+}} \frac{x_{1}^{+} x_{2}^{-}-1}{x_{1}^{-} x_{2}^{-}-1}, \\
& \mathcal{R}^{\alpha}{ }_{\beta}{ }^{\gamma}{ }_{\delta}=\frac{U_{2}}{U_{1}} \frac{x_{1}^{+}-x_{2}^{-}}{x_{1}^{-}-x_{2}^{+}}\left[\delta_{\delta}^{\alpha} \delta_{\beta}^{\gamma}+\left(\delta_{\beta}^{\alpha} \delta_{\delta}^{\gamma}-\delta_{\delta}^{\alpha} \delta_{\beta}^{\gamma}\right) \frac{x_{1}^{+}-x_{2}^{+}}{x_{1}^{+}-x_{2}^{-}} \frac{x_{2}^{-}}{x_{2}^{+}} \frac{x_{1}^{-} x_{2}^{+}-1}{x_{1}^{-} x_{2}^{-}-1}\right], \\
& \mathcal{R}^{a}{ }_{\alpha \beta}{ }^{b}{ }_{\beta}=-\varepsilon^{a b} \varepsilon_{\alpha \beta} \frac{\gamma_{1} \gamma_{2} U_{2} x_{1}^{-} x_{2}^{-}\left(x_{1}^{+}-x_{2}^{+}\right)}{x_{1}^{+} x_{2}^{+}\left(x_{1}^{-} x_{2}^{-}-1\right)\left(x_{2}^{+}-x_{1}^{-}\right)}, \\
& \mathcal{R}^{\alpha{ }^{\beta}{ }_{b}{ }_{b}}=\varepsilon_{a b} \varepsilon^{\alpha \beta} \frac{\left(x_{1}^{+}-x_{2}^{+}\right)\left(x_{1}^{-}-x_{1}^{+}\right)\left(x_{2}^{-}-x_{2}^{+}\right)}{\gamma_{1} \gamma_{2} U_{1}\left(x_{1}^{-} x_{2}^{-}-1\right)\left(x_{2}^{+}-x_{1}^{-}\right)}
\end{aligned}
$$

and

$$
\begin{aligned}
\mathcal{R}^{a}{ }_{b}{ }^{\alpha}{ }_{\beta} & =\delta_{b}^{a} \delta_{\beta}^{\alpha} \frac{1}{U_{1}} \frac{x_{1}^{+}-x_{2}^{+}}{x_{1}^{-}-x_{2}^{+}}, & \mathcal{R}^{a}{ }_{\beta}{ }^{\alpha}{ }_{b} & =\delta_{b}^{a} \delta_{\beta}^{\alpha} \frac{U_{2}}{U_{1}} \frac{x_{2}^{-}-x_{2}^{+}}{x_{2}^{+}-x_{1}^{-}} \frac{\gamma_{1}}{\gamma_{2}}, \\
\mathcal{R}^{\alpha}{ }_{b}{ }^{a}{ }_{\beta} & =\delta_{b}^{a} \delta_{\beta}^{\alpha} \frac{x_{1}^{+}-x_{1}^{-}}{x_{2}^{+}-x_{1}^{-}} \frac{\gamma_{2}}{\gamma_{1}}, & \mathcal{R}^{\alpha}{ }_{\beta}{ }^{a}{ }_{b} & =\delta_{b}^{a} \delta_{\beta}^{\alpha} U_{2} \frac{x_{1}^{-}-x_{2}^{-}}{x_{1}^{-}-x_{2}^{+}} .
\end{aligned}
$$

The parameters $x_{1,2}^{ \pm}$are related to the spectral parameters $u_{1,2}$, respectively in the following way

$$
u=x^{+}+\frac{1}{x^{+}}-\frac{\hbar}{2}=x^{-}+\frac{1}{x^{-}}+\frac{\hbar}{2} .
$$

The parameter $\hbar$ corresponds to the coupling constant and the parameters $U$ are related to the above by $U^{2}=x^{+} / x^{-}$. Finally, the additional parameter $\gamma$ defines the relative normalization of bosons $(\phi)$ and fermions $(\psi)$. The R-matrix is the intertwiner of the centrally extended $\mathfrak{s u}(2 \mid 2)$ superalgebra. This algebra contains two $\mathfrak{s u}(2)$ subalgebras that act on the bosons and fermions respectively. The R-matrix is a symmetric matrix with respect to an appropriately chosen inner product for the states

$$
\left\langle\phi^{a} \mid \phi^{b}\right\rangle=\delta^{a b}, \quad\left\langle\psi^{\alpha} \mid \psi^{\beta}\right\rangle=\delta^{\alpha \beta} \frac{U}{\gamma^{2}}\left(x^{+}-x^{-}\right)=\delta^{\alpha \beta} \frac{x^{+}}{\gamma^{2}}\left(U-U^{-1}\right) .
$$




\subsection{Symmetric states}

Let us first consider the points where the R-matrix becomes of rank 8. They correspond to the two special points $x_{1}^{+}=x_{2}^{-}$(corresponding to $u_{1}=u_{2}+\hbar$ ) as well as $x_{1}^{-}=x_{2}^{+}$ (corresponding to $u_{1}=u_{2}-\hbar$ ). In the following we will consider the point $x_{1}^{+}=x_{2}^{-}$. The considerations for the other point are completely analogous.

This first ingredient we need is the matrix of normalized eigenvectors $\mathcal{E}$ of the fundamental R-matrix (5.1). There are four bosonic vectors $\left|S^{(a b)}\right\rangle$ and $\left|S^{[34]}\right\rangle$. Three of them have unit eigenvalue $\lambda_{(a b)}=1$ and form a standard triplet under the bosonic $\mathfrak{s u}(2)$

$$
\left|\mathrm{S}^{(a a)}\right\rangle=\left|\phi^{a}\right\rangle \otimes\left|\phi^{a}\right\rangle, \quad \quad\left|\mathrm{S}^{(12)}\right\rangle=\frac{1}{\sqrt{2}}\left(\left|\phi^{1}\right\rangle \otimes\left|\phi^{2}\right\rangle+\left|\phi^{2}\right\rangle \otimes\left|\phi^{1}\right\rangle\right),
$$

and the fourth contains two fermionic states and is a singlet under both $\mathfrak{s u}(2)$ 's

$$
\begin{aligned}
\left|\mathrm{S}^{[34]}\right\rangle & =\frac{\gamma_{1} \gamma_{2} U_{2}^{-1}\left[\varepsilon^{\alpha \beta}\left|\psi^{\alpha}\right\rangle \otimes\left|\psi^{\beta}\right\rangle\right]+\left(1-\frac{1}{2}\left(U_{2}^{-2}+U_{1}^{2}\right)\right)\left[\varepsilon^{a b}\left|\phi^{a}\right\rangle \otimes\left|\phi^{b}\right\rangle\right]}{\sqrt{2} \sqrt{U_{1} U_{2}^{-1}\left(x_{2}^{+}-x_{2}^{-}\right)\left(x_{1}^{+}-x_{1}^{-}\right)+\left(1-\frac{1}{2}\left(U_{2}^{-2}+U_{1}^{2}\right)\right)^{2}}} \\
\lambda_{[34]} & =\frac{x_{2}^{+}-x_{2}^{-}}{1-x_{1}^{+} x_{1}^{-}}\left[\frac{x_{2}^{-}-x_{1}^{-}}{1-x_{1}^{+} x_{2}^{+}}+\frac{2}{U_{1} U_{2}} \frac{1-x_{1}^{-} x_{2}^{+}}{x_{2}^{+}-x_{1}^{-}}\right] .
\end{aligned}
$$

There are four fermionic eigenstates $\left|\mathrm{S}^{a \alpha}\right\rangle$

$$
\left|S^{a \alpha}\right\rangle=\frac{\gamma_{2}\left|\phi^{a}\right\rangle \otimes\left|\psi^{\alpha}\right\rangle+\gamma_{1} U_{2}\left|\psi^{\alpha}\right\rangle \otimes\left|\phi^{a}\right\rangle}{\sqrt{U_{2}\left(x_{2}^{+}-x_{2}^{-}\right)+U_{1} U_{2}^{2}\left(x_{1}^{+}-x_{1}^{-}\right)}}, \quad \lambda_{a \alpha}=\frac{1}{U_{2}} \frac{x_{2}^{-}-x_{2}^{+}}{x_{1}^{-}-x_{2}^{+}}+U_{1} \frac{x_{1}^{-}-x_{1}^{+}}{x_{1}^{-}-x_{2}^{+}} .
$$

After packaging these vectors in the matrix $\mathcal{E}$ and taking $\mathcal{F}=\mathcal{E}^{\top}$, it is readily checked that $(2.10)$ and $(2.14)$ hold.

We are now in a position to apply our fusion procedure. Let us consider the fused matrix (2.15) and make it symmetric by applying the transformation $W$ as in (3.12). It is then straight-forward to check that this R-matrix coincides with $\mathbb{S}^{B A}$ from $[9]$.

As a non-trivial example let us compute two scattering processes

$$
\mathcal{R}^{\prime B A}\left(u_{\langle 12\rangle}, u_{3}\right)\left|\mathrm{S}^{(11)}\right\rangle \otimes\left|\phi^{1}\right\rangle, \quad \quad \mathcal{R}^{\prime B A}\left(u_{\langle 12\rangle}, u_{3}\right)\left|\mathrm{S}^{13}\right\rangle \otimes\left|\psi^{3}\right\rangle,
$$

It is easy to see that

$$
\mathcal{R}^{\prime B A}\left(u_{\langle 12\rangle}, u_{3}\right)\left|\mathrm{S}^{(11)}\right\rangle \otimes\left|\phi^{1}\right\rangle=\left|\mathrm{S}^{(11)}\right\rangle \otimes\left|\phi^{1}\right\rangle,
$$

which agrees with $a_{1}=1$ from section 6.1 .2 of $[9]$. This shows that we have the same normalization for the bound state S-matrix. Subsequently, we have by definition

$$
\begin{aligned}
& \mathcal{R}^{\prime B A}\left|\mathrm{~S}^{13}\right\rangle \otimes\left|\psi^{3}\right\rangle \\
= & W \mathcal{F}_{12} \mathcal{R}_{13} \mathcal{R}_{23} \mathcal{E}_{12} W^{-1}\left|\mathrm{~S}^{13}\right\rangle \otimes\left|\psi^{3}\right\rangle \\
= & {\left[\frac{U_{2}\left(x_{1}^{+}-x_{1}^{-}\right)\left(x_{2}^{+}-x_{3}^{+}\right)\left(x_{3}^{-}-x_{1}^{+}\right)}{\left(x_{1}^{-}-x_{3}^{+}\right)\left(x_{3}^{+}-x_{2}^{-}\right)}+\frac{\left(x_{2}^{+}-x_{2}^{-}\right)\left(x_{3}^{+}-x_{1}^{+}\right)\left(x_{3}^{-}-x_{2}^{+}\right)}{U_{1}\left(x_{1}^{-}-x_{3}^{+}\right)\left(x_{2}^{-}-x_{3}^{+}\right)}\right.} \\
& \left.\quad+\frac{U_{2}\left(x_{2}^{+}-x_{2}^{-}\right)\left(x_{1}^{-}-x_{1}^{+}\right)\left(x_{3}^{-}-x_{3}^{+}\right)}{\left(x_{1}^{-}-x_{3}^{+}\right)\left(x_{3}^{+}-x_{2}^{-}\right)}\right] \\
& \quad \frac{U_{3}}{U_{2}\left(x_{2}^{+}-x_{2}^{-}\right)+U_{1} U_{2}^{2}\left(x_{1}^{+}-x_{1}^{-}\right)}\left|\mathrm{S}^{13}\right\rangle \otimes\left|\psi^{3}\right\rangle \\
= & \frac{U_{3}}{U_{1} U_{2}} \frac{x_{2}^{+}-x_{3}^{-}}{x_{1}^{-}-x_{3}^{+}}\left|\mathrm{S}^{13}\right\rangle \otimes\left|\psi^{3}\right\rangle,
\end{aligned}
$$


where we used the inner product $(5.5)$ and $x_{1}^{+}=x_{2}^{-}$. This result exactly coincides with the literature, in particular it is the coefficient $a_{7}$ from section 6.1 .2 of $[9]$. In fact it is not hard to check that the representation $(3.2)$ is exactly the two particle bound state representation from $[9]$.

Let us conclude this section by considering the complementary fusion procedure. The complement is spanned by antisymmetric states $|\mathrm{A}\rangle=\left\{|\mathrm{A}\rangle^{(\alpha \beta)},\left|\mathrm{A}^{[12]}\right\rangle,\left|\mathrm{A}^{a \alpha}\right\rangle\right\}$ that are perpendicular to the symmetric states $|S\rangle$ given above.

For these states, one can then easily compute the complementary R-matrix (3.18). In particular, due to the upper triangular structure $(3.22)$, the computation simplifies somewhat. For instance, let us consider the analogue of the states (5.9)

$$
\left|\mathrm{A}^{(33)}\right\rangle \otimes\left|\psi^{3}\right\rangle, \quad\left|\mathrm{A}^{13}\right\rangle \otimes\left|\phi^{1}\right\rangle .
$$

The action of $\mathcal{R}_{13} \mathcal{R}_{23}$ on both states is simply multiplicative and yields

$$
\begin{aligned}
\mathcal{R}_{13} \mathcal{R}_{23}\left|\mathrm{~A}^{(33)}\right\rangle \otimes\left|\psi^{3}\right\rangle & =\frac{x_{1}^{+}-x_{3}^{-}}{x_{1}^{-}-x_{3}^{+}} \frac{U_{3}}{U_{1}} \cdot \frac{x_{2}^{+}-x_{3}^{-}}{x_{2}^{-}-x_{3}^{+}} \frac{U_{3}}{U_{2}}\left|\mathrm{~A}^{(33)}\right\rangle \otimes\left|\psi^{3}\right\rangle, \\
\mathcal{R}_{13} \mathcal{R}_{23}\left|\mathrm{~A}^{13}\right\rangle \otimes\left|\phi^{1}\right\rangle & =\frac{x_{1}^{+}-x_{3}^{-}}{x_{1}^{-}-x_{3}^{+}} \frac{U_{3}}{U_{1}} \cdot \frac{x_{2}^{+}-x_{3}^{-}}{x_{2}^{-}-x_{3}^{+}} \frac{U_{3}}{U_{2}} \cdot \frac{U_{1} U_{2}}{U_{3}} \frac{x_{1}^{-}-x_{3}^{+}}{x_{2}^{+}-x_{3}^{-}}\left|\mathrm{A}^{13}\right\rangle \otimes\left|\phi^{1}\right\rangle .
\end{aligned}
$$

This then corresponds to the fused R-matrix on antisymmetric states corresponding to the other point of lower rank $x_{1}^{-}=x_{2}^{+}$. Notice that the matrix has a different normalization and the inverse of the coefficient (5.11) appears, which is in agreement with [12] where the relation between the symmetric and antisymmetric R-matrices is discussed.

\subsection{Singlet state}

The R-matrix coefficients in (5.2) have a common factor of $\left(x_{1}^{+}-x_{2}^{+}\right) /\left(x_{1}^{-} x_{2}^{-}-1\right)$, which has a potential singularity at $x_{2}^{-}=1 / x_{1}^{-} \cdot 7$ Furthermore, $x_{2}^{+} \neq x_{1}^{+}$in order for the numerator to be non-zero, i.e. $x_{2}^{+}=1 / x_{1}^{+}$. The singularity affects only the $\mathfrak{s u}(2) \times \mathfrak{s u}(2)$ singlet sector spanned by the two states

$$
|\mathrm{BB}\rangle=\varepsilon^{a b} \phi^{a} \otimes \phi^{b}, \quad|\mathrm{FF}\rangle=\varepsilon^{\alpha \beta} \psi^{\alpha} \otimes \psi^{\beta} .
$$

The action on the remaining 14 states is finite. Acting on the singlet, the $\mathrm{R}$-matrix reduces to a $2 \times 2$ matrix $M$. In the limit $x_{2}^{ \pm} \rightarrow 1 / x_{1}^{ \pm}$, it has the following singularity structure

$$
M=\frac{1}{\epsilon} M^{(-1)}+M^{(0)}+\ldots
$$

Up to an overall factor we find for the residue

$$
M^{(-1)} \sim\left(\begin{array}{cc}
1 & -\left(U_{1}-U_{1}^{-1}\right) / \gamma_{1} \gamma_{2} \\
U_{1} U_{2} \gamma_{1} \gamma_{2} /\left(U_{1}-U_{1}^{-1}\right) & -U_{1} U_{2}
\end{array}\right)
$$

We know that $U^{2}=x^{+} / x^{-}$and hence $U_{1} U_{2}= \pm 1$. Both values of the latter signs are permitted, and we have to discuss the two cases separately, as they lead to rather distinct behavior.

\footnotetext{
${ }^{7}$ Here, we do not explicitly multiply the R-matrix by an overall factor to compensate the singularity. Hence we will consider the most singular contributions to the matrix.
} 
We start with $U_{1} U_{2}=-1$ which is analogous to the cases discussed above. There are two eigenvectors

$$
\left(U_{1}-U_{1}^{-1}\right)|\mathrm{BB}\rangle+\gamma_{1} \gamma_{2}|\mathrm{FF}\rangle \quad \text { and } \quad\left(U_{1}-U_{1}^{-1}\right)|\mathrm{BB}\rangle-\gamma_{1} \gamma_{2}|\mathrm{FF}\rangle .
$$

The eigenvalues are 2 and 0 , respectively, therefore only the former state is singular. It is a singlet of the Yangian algebra, however it has a non-trivial charge $U_{\langle 12\rangle}=U_{1} U_{2}=-1$ which plays a role for the coproduct. The other state belongs to an adjoint representation of $\mathfrak{p s u}(2 \mid 2) \cdot{ }^{8}$ The singularity structure of this case is peculiar with respect to complementary fusion: In the limit $x_{2}^{ \pm} \rightarrow 1 / x_{1}^{ \pm}$, the eigenvalue of the above non-singular singlet state approaches zero even faster than for the 14 non-singlet states. We find

$$
\lambda_{1 \mathrm{a}} \sim \frac{1}{\epsilon}, \quad \lambda_{14} \sim 1, \quad \lambda_{1 \mathrm{~b}} \sim \epsilon .
$$

This means that complementary fusion based on $\mathcal{R}_{21}$ produces merely one composite state rather than 15 . This state is just the other singlet, and in fact one can see that exchanging the two sites in (5.18) interchanges the two states. ${ }^{9}$

The other case $U_{\langle 12\rangle}=U_{1} U_{2}=+1$ has only one eigenvector

$$
\left(U_{1}-U_{1}^{-1}\right)|\mathrm{BB}\rangle+\gamma_{1} \gamma_{2}|\mathrm{FF}\rangle,
$$

and its eigenvalue is 0 . The matrix $M^{(-1)}$ does not admit a second eigenvector because it has a non-trivial Jordan decomposition. This makes the case very special, and the fusion procedure described in this paper does not immediately apply. Let us therefore try to understand what is going on: The eigenstate is the singlet state discussed in [4]. There it was shown that the state behaves just like a fused state under scattering with other states, i.e. it preserves its form. To understand the role of the other singlet state, it makes sense to take a closer look at the limit $x_{2}^{ \pm} \rightarrow 1 / x_{1}^{ \pm}$. Here, both singlet eigenvalues remain finite, whereas the eigenvectors become collinear. Therefore, at $x_{2}^{ \pm}=1 / x_{1}^{ \pm}$there is only one meaningful eigenvector, the difference of the eigenvectors plays no significant role. Even though all eigenvalues remain finite at this point, fusion does take place due to the coincidence of eigenvectors.

The fused R-matrix $\mathcal{R}_{\langle 12\rangle 3}$ of the singlet state with $U_{\langle 12\rangle}=+1$ was shown to be trivial in [4] up to an overall phase factor related to crossing symmetry [13]. Based on this result one can easily derive the fused R-matrix of the singlet state with $U_{\langle 12\rangle}=-1$. The point is that the factor $U_{1}$ appears in odd powers in (5.2) and (5.3) only when the third index is fermionic. Flipping the sign $U_{2}$ flips the sign of $\mathcal{R}_{\langle 12\rangle 3}$ precisely if state 3 is fermionic (5.1). This means that the state with $U_{\langle 12\rangle}=-1$ behaves like a fermionic singlet, while $U_{\langle 12\rangle}=+1$ corresponds to a bosonic singlet. This observation is in line with the coproduct rule of odd generators.

\section{Acknowledgements}

We would like to thank G. Arutyunov, S. Frolov, J. Myers and A. Torrielli for discussions. The work of NB and MdL is partially supported by grant no. 200021-137616 from the Swiss National Science Foundation and through the NCCR SwissMAP. The work of NB is partially supported by grant no. 615203 from the European Research Council under the FP7. MdL was also supported by FNU through grant number DFF132300082.

\footnotetext{
${ }^{8}$ The adjoint is reducible but indecomposable, and the former singlet also acts as the top components of this representation.

${ }^{9}$ One has to take into account the sign from exchanging fermions and that $U_{1} \rightarrow-U_{1}^{-1}$.
} 


\section{References}

[1] R. J. Baxter, "Exactly solved models in statistical mechanics", Academic Press (1982), London, UK.

[2] M. Karowski, "On the Bound State Problem in (1+1)-dimensional Field Theories", Nucl. Phys. B153, 244 (1979). • P. P. Kulish, N. Yu. Reshetikhin and E. K. Sklyanin, "Yang-Baxter Equation and Representation Theory. 1.", Lett. Math. Phys. 5, 393 (1981). - M. Jimbo, "A q difference analog of $U(g)$ and the Yang-Baxter equation", Lett. Math. Phys. 10, 63 (1985). • M. Jimbo, "A q Analog of $U(g l(n+1))$, Hecke Algebra and the Yang-Baxter Equation", Lett. Math. Phys. 11, 247 (1986). • A. N. Kirillov and N. Yu. Reshetikhin, "Exact solution of the integrable XXZ Heisenberg model with arbitrary spin. I. The ground state and the excitation spectrum",

J. Phys. A20, 1565 (1987) • L. Mezincescu and R. I. Nepomechie, "Fusion procedure for open chains", J. Phys. A25, 2533 (1992).

[3] N. Beisert et al., "Review of AdS/CFT Integrability: An Overview", Lett. Math. Phys. 99, 3 (2012), arxiv:1012.3982.

[4] N. Beisert, "The SU(2/2) dynamic S-matrix", Adv. Theor. Math. Phys. 12, 945 (2008), hep-th/0511082.

[5] G. Arutyunov, S. Frolov, J. Plefka and M. Zamaklar, "The Off-Shell Symmetry Algebra of the Light-Cone $A d S_{5} \times S^{5}$ ", J. Phys. A40, 3583 (2007), hep-th/0609157. • N. Beisert, "The S-matrix of AdS / CFT and Yangian symmetry", PoS SOLVAY, 002 (2006), arxiv:0704.0400.

[6] B. S. Shastry, "Decorated star-triangle relations and exact integrability of the one-dimensional Hubbard model", J. Stat. Phys. 50, 57 (1988).

[7] N. Beisert, "The Analytic Bethe Ansatz for a Chain with Centrally Extended su(2/2) Symmetry", J. Stat. Mech. 07, P01017 (2007), nlin/0610017. • M. Martins and C. Melo, "The Bethe ansatz approach for factorizable centrally extended S-matrices", Nucl. Phys. B785, 246 (2007), hep-th/0703086.

[8] N. Dorey, "Magnon Bound States and the AdS/CFT Correspondence", J. Phys. A39, 13119 (2006), hep-th/0604175. • H.-Y. Chen, N. Dorey and K. Okamura, "Dyonic giant magnons", JHEP 0609, 024 (2006), hep-th/0605155. • G. Arutyunov and S. Frolov, "On String S-matrix, Bound States and TBA", JHEP 0712, 024 (2007), arxiv:0710.1568.

[9] G. Arutyunov and S. Frolov, "The S-matrix of String Bound States", Nucl. Phys. B804, 90 (2008), arxiv:0803.4323.

[10] M. de Leeuw, "Bound States, Yangian Symmetry and Classical r-matrix for the AdS $S_{5} \times$ $S^{5}$ Superstring", JHEP 0806, 085 (2008), arxiv:0804.1047. • G. Arutyunov, M. de Leeuw and A. Torrielli, "The Bound State S-Matrix for AdS $S_{5} \times S^{5}$ Superstring", Nucl. Phys. B819, 319 (2009), arxiv:0902.0183.

[11] G. Arutyunov, S. Frolov and M. Zamaklar, "The Zamolodchikov-Faddeev algebra for AdS $S_{5}$ $\times S^{5}$ superstring", JHEP 0704, 002 (2007), hep-th/0612229.

[12] Z. Bajnok and R. A. Janik, "Four-loop perturbative Konishi from strings and finite size effects for multiparticle states", Nucl. Phys. B807, 625 (2009), arxiv:0807.0399.

[13] R. A. Janik, "The $A d S_{5} \times S^{5}$ superstring worldsheet S-matrix and crossing symmetry", Phys. Rev. D73, 086006 (2006), hep-th/0603038. 